Revista de Matemática: Teoría y Aplicaciones 2002 9(2) : 59-74

CIMPA - UCR - CCSS ISSN: 1409-2433

\title{
APLiCACIÓN DEL MÉTODO DE SELECCIÓN DE PROPUESTAS A LA DESCOMPOSICIÓN DE TAREAS DISCRETAS DE OPTIMIZACIÓN DE GRAN COMPLEJIDAD
}

\author{
José Arzola Ruiz* Alberto Fiol Zulueta ${ }^{* *}$
}

Recibido: 12 Sep 2000

\begin{abstract}
Resumen
En el artículo se estudia el método de Selección de Propuestas y su generalización, así como el problema de la organización de estructuras de conciliación de decisiones entre sistemas interelacionados, se brindan enfoques para la conciliación óptima de decisiones entre sistemas organizados en estructuras jerárquicas, coincidentes con los procedimientos de composición de decisiones de las subtareas resultantes de la descomposición de tareas de optimización de gran complejidad y se aplica el método de Selección de Propuestas a la solución de este problema.
\end{abstract}

La exposición se organiza en las siguientes etapas:

- Tarea de selección de propuestas, su generalización, métodos de solución y esquemas de descomposición de tareas de gran complejidad.

- Enfoques para la conciliación de decisiones entre sistemas organizados en diferentes estructuras.

Palabras clave: Optimización discreta, descomposición de tareas, conciliación de decisiones, preparacin y toma de decisiones, teoría de sistemas.

\begin{abstract}
In the paper the method of Selection of Proposals and it generalisation, the problem of the organization of structures of conciliating decisions among interrelated systems are studied. Focuses are offered for the optimal decisions conciliating among systems organized in hierarchical structures, coincident with the procedures of composition of decisions of resulting subtask from the decomposition of great complexity optimization tasks and the method of Selection of Proposals is applied to the solution of this problem.

The contents are organized as follows:
\end{abstract}

\footnotetext{
${ }^{*}$ Unidad Docente Metalúrgica, Facultad de Ingeniería Mecánica Instituto Superior Politécnico "José Antonio Echeverría" La Habana, Cuba. E-Mail: udm@aacero.colombus.cu

** Departamento de Matem'aticas, Facultad de Ingeniería, Instituto Superior Politécnico “José Antonio Echeverría", La Habana, Cuba.
} 
- Selection of proposals task, its generalization, solution methods and schemes of decomposition of great complexity tasks.

- Approaches for decisions conciliating among systems organized in different structures.

Keywords: Discrete optimization, task decomposition, conciliating decisions, decision taking, system theory.

Mathematics Subject Classification: 90B50

\section{La tarea de selección de propuestas, su generalización, méto- dos de solución y esquemas de descomposición de tareas de gran complejidad}

Uno de los problemas fundamentales de la optimización descreta consiste en la descomposición de tareas de gran complejidad en subtareas más sencillas. Independientemente de las especificidades de cada tarea compleja de optimización concreta, un esquema de descomposición puede consistir en la generación, para cada subtarea, de series de soluciones locales y la búsqueda posterior de solución a la tarea original entre las series de soluciones encontradas para las diferentes subtareas. El método de Selección de Propuestas está concebido, entre otros objetivos, para enfrentar el problema de composición de soluciones de tareas de optimización discreta resultantes de la descomposición, posibilitando la obtención de solución a la tarea original (Arzola, 1989). Asociado al método fue formulada la siguienteTarea de Selección de Propuestas.

Sea cada elemento del conjunto finito $S=(1,2, \ldots, s, \ldots, n)$ puesto en correspondencia con una serie ordenada, monótona creciente de números reales:

$$
Z_{1}^{s}<Z_{2}^{s}<\ldots<Z_{i}^{s}<\ldots<Z_{i}^{s} \in \mathbb{R}
$$

La serie (1) es caracterizada por el conjunto de índices $I_{s}=(1,2, \ldots, i, \ldots)$ de sus elementos.

Las combinaciones posibles de índices se determinan por los elementos

$$
e^{k}=\left(k_{1}, k_{2}, \ldots, k_{s}, \ldots, k_{n}\right)
$$

del producto de conjuntos:

$$
E=I_{1} \times I_{2} \times \ldots \times I_{n}
$$

Se supone que para cada $e^{k} \in E$, se puede obtener

$$
\varepsilon\left(e^{k}\right) \geq 0
$$

Se requiere hallar el elemento $e \in E$ que minimice la función:

$$
Z\left(e^{k}\right)=\sum_{s=1}^{n} Z_{k}^{s}+\varepsilon\left(e^{k}\right) / e^{k} \in E
$$


A partir de la formulación realizada fueron estudiadas las propiedades de esta tarea y elaborado el método exacto de solución, el que se expone en el libro Sistemas de Ingeniería. En ese mismo libro se desarrolla el tema de la descomposición de tareas complejas de optimización discreta con utilización del método de Selección de Propuestas.

Definiciones básicas:

- Propuestas de $s$ : son los elementos del conjunto $I_{s}$.

- Valoraciones locales o pérdidas locales: son los elementos de la serie (1), que se simbolizarán como $Z_{k}^{s}$

- Función de pérdidas sistémicas: es la función $\varepsilon\left(e^{k}\right)$.

- Función de pérdidas totales: es la función $Z\left(e^{k}\right)$.

- Relaciones entre los elementos del conjunto $E$ :

$$
\begin{aligned}
& e^{r} \geq e^{m} / r_{s} \geq m_{s}, \forall s \in S \\
& e^{r}=e^{m} / r_{s}=m_{s}, \forall s \in S \\
& e^{r} \leq e^{m} / r_{s} \leq m_{s}, \forall s \in S
\end{aligned}
$$

- El conjunto $E_{k} \subset E$ se denomina conjunto representado por el elemento $e^{k}$ si para cualquier $e^{r} \in E_{k}, e^{r} \geq e^{k}$.

- El conjunto $E_{k}^{\prime}$ representado por el elemento $e^{m}$ se denomina conjunto sucesor de $E_{k}$ por el elemento $r$ si $m_{s}=k_{s}, s \neq r$ y $r$ y $m_{r}=k_{r}+1$. El conjunto $E_{k}$ se denomina conjunto antecesor de $E_{k}^{\prime}$ por el elemento $r$ de $S$. Por definición:

$$
E_{k}^{\prime} \subset E_{k} \subset E
$$

Definición 1 Sea la tarea de optimización

$$
Z^{o p t}=\min \{Z(x) / x \in D\}
$$

el espacio de soluciones $D^{\alpha} \subset D$ se denomina espacio de soluciones $\alpha$-óptimo y la solución $x^{i} \in D$ solución $\alpha$-óptima si

$$
x^{i} \in D^{\alpha} \Leftrightarrow Z\left(x^{i}\right)-Z^{o p t} \leq \alpha
$$

\section{Propiedades fundamentales}

Teorema 2 La condición suficiente para que ningún elemento del conjunto $E_{k} \subset E$ representado por el elemento $e^{k}$ sea solución de la tarea (4), es la existencia de un elemento $e^{r} \notin E_{k}$ para el cual se cumpla la condición:

$$
\sum_{s} Z_{k}^{s}>\sum_{s} Z_{r}^{s}+\varepsilon\left(e^{r}\right)
$$


Teorema 3 Sea para el elemento $e^{m} \in E$ conocido un valor acotado de $\varepsilon\left(e^{m}\right)$ y para cada $s \in S$ se puede hallar un indice finito $i_{s}$ para el cual:

$$
Z_{i_{s}}^{s}-Z_{1}^{s}>\varepsilon\left(e^{m}\right)+\sum_{r \in S}\left(Z_{m}^{r}-Z_{1}^{r}\right)
$$

entonces, para cualquier componente de la solución óptima de la tarea ept se cumple:

$$
i_{s}^{o p t}<i_{s}
$$

Consecuencia 1 Al evaluar las pérdidas sistémicas correspondientes a una combinación determinada, puede ser excluida la consideración de todas aquellas combinaciones, cuyos componentes por el elemento $s$ superen el valor $i_{s}$, siempre que este indice satisfaga la expresión (6).

Consecuencia 2 Si existe al menos una solución de la tarea (4) en lugar de las series infinitas (1), es suficiente la consideración de series finitas dadas por los conjuntos de indices $I_{s}=\left(1,2, \ldots, i_{s}\right)$, tales que $i_{s}^{\text {opt }}<i_{s}$.

Teorema 4 Sea el subconjunto $E^{*}$ formado por las combinaciones posibles de indices:

$$
i / i \in \bar{I}_{s}=\left(1,2, \ldots, i_{s}\right), Z_{i_{s}}^{s}-Z_{1}^{s} \leq \alpha ; \forall s \in S, \alpha \in \mathbb{R}^{*} .
$$

entonces, si $Z\left(e^{m}\right)$ es solución de la tarea en el conjunto $E^{*}$ y $Z\left(e^{o p t}\right)$ el mínimo global en el conjunto $E$ y $e^{m} \neq e^{o p t}$ tiene lugar:

$$
Z\left(e^{m}\right)-Z\left(e^{o p t}\right) \leq \varepsilon\left(e^{m}\right)+\sum_{s}\left(Z_{m}^{s}-Z_{1}^{s}\right)-\operatorname{máx}_{s}\left(Z_{i_{s}}^{s}-Z_{1}^{s}\right)
$$

El teorema 1 permite eliminar conjuntos completos en el proceso de la búsqueda de la solución óptima, pudiéndose no considerarse los conjuntos representados por los elementos $e^{k}$ del conjunto $E$ donde se cumple la condición (5).

El teorema 2 facilita el restringir el conjunto de propuestas $I_{s}$ en la medida que se van obteniendo las pérdidas sistémicas para las diferentes soluciones del método de selección de propuestas. Además, es posible concluir que para todo sistema existe un valor $\alpha$, el cual es común para todos sus elementos y cada uno de ellos, de modo que la solución óptima para todo el sistema se encuentra entre las soluciones $\alpha$-óptimas de sus componentes.

El parámetro $\alpha$ se calcula mediante:

$$
\alpha=\varepsilon\left(e^{m}\right)+\sum_{r}\left(Z_{m}^{r}-Z_{1}^{r}\right) .
$$

La fórmula anterior expresa las pérdidas totales por concepto de interacción entre los elementos del sistema y por no poderse adoptar las soluciones óptimas locales por los diferentes elementos del sistema. Es evidente que el valor de $\alpha$ no es posible calcularlo directamente, pues no son conocidas inicialmente las soluciones $e^{m}$, lo que trae como consecuencia que su valor sea posible estimarlo a partir de información estadística acumulada anteriormente.

El teorema 3 permite el cálculo de la precisión alcanzada por una solución intermedia cualquiera; es bueno destacar que en ocasiones no es importante el cálculo de la solución óptima y basta con obtener una solución lo suficientemente cercana a la óptima. 


\section{Algoritmo de la selección óptima}

Se construye un árbol cuyo tronco contiene a todo el conjunto $E$, siendo su representante el elemento $e^{1}=(1,1, \ldots, 1)$.

La cota inferior del tronco es:

$$
V\left(e^{1}\right)=\sum_{s} Z_{1}^{s}
$$

El conjunto $E$ tiene $n$ subconjuntos sucesores por los correspondientes elementos $s \in S$, por lo que:

$$
E-e^{1}=\bigcup_{s} E_{s}^{\prime}
$$

El primer nivel de ramificación incluye $n$ nodos, correspondientes a los subconjuntos sucesores $E_{s}^{\prime}$. Si la valoración del nodo $r$ :

$$
V\left(e^{r}\right)=Z_{2}^{r}+\sum_{s \neq r} Z_{1}^{s}
$$

es el superior al valor $Z\left(e^{1}\right)$, entonces este nodo y el conjunto de elementos a él asociado no se contemplan en lo adelante. Se calcula el valor de la función (4) para el elemento representante del subconjunto asociado al nodo con valoración inferior mínima y si éste es menor que $Z\left(e^{1}\right)$ se adopta el nuevo valor record.

El nuevo valor se compara con las cotas inferiores de los nodos en espera, eliminándose los nodos que satisfacen la condición (5). El nodo con cota inferior mínima se somete a ramificación en los subcojuntos sucesores del conjunto a él asociado, se rectifica el valor record y se verifica la posibilidad de eliminar parte de los nodos de espera.

Este procedimiento se repite mientras quedan nodos en espera y la solución óptima es aquella correspondiente a la combinación representante del nodo ramificado con valor mínimo del funcional (4).

Se destaca como diferencia esencial del método de Selección de Propuestas con respecto al método de ramificaciones y acotaciones la partición del conjunto original de variantes en subconjuntos no interceptados, por lo que la cantidad de nodos en los niveles sucesivos de la ramificación crece más lentamente que el exponente de $m$, siendo $m$ el consecutivo del nivel de ramificación.

\section{Generación de soluciones $\alpha$-óptimas}

Debido a que en ocasiones la tarea a solucionar forma parte de una tarea más compleja o se necesita generar una serie de opciones diferentes, entonces se precisa generar soluciones $\alpha$-óptimas de la tarea de la selección de propuestas.

Lo anterior puede ser logrado mediante la introducción de determinadas adecuaciones al algoritmo de selección óptima, como son:

- rectificación de las condiciones de eliminación de nodos en espera,

- limitación de las propuestas locales,

- verificación de la precisión. 
De ahí que, en vez de satisfacer (2.6) se requiere el cumplimiento de la condición:

$$
\sum_{s} Z_{k}^{s}>\sum_{s} Z_{r}^{s}+\varepsilon\left(e^{r}\right)+\alpha
$$

Ahora, en vez de (2.9), se debe cumplir:

$$
Z_{i_{s}}^{s}-Z_{1}^{s}>\varepsilon\left(e^{r}\right)+\sum_{s}\left(Z_{i}^{s}-Z_{1}^{s}\right)+\alpha
$$

La valoración de la precisión alcanzada se realiza mediante:

$$
Z\left(e^{m, \alpha}\right)-Z\left(e^{o p t, \alpha}\right) \leq \varepsilon\left(e^{m, \alpha}\right)+\sum_{s}\left(Z_{m, \alpha}^{s}-Z_{1}^{s}\right)-\operatorname{máx}\left(Z_{i_{s}}^{s}-Z_{1}^{s}\right)
$$

donde:

$e^{m, \alpha}$ : combinación representante del nodo ramificado con el peor valor de la función (4), entre las que se diferencian del óptimo en no más que $\alpha$ en el conjunto $E$.

$e^{o p t, \alpha}$ : peor entre las soluciones $\alpha$-óptimas en todo el conjunto $E$.

$Z_{m, \alpha}^{s}$ : valor de la función de pérdidas locales correspondiente a la componente $s$ de la combinación $e^{m, \alpha}$.

A partir de la expresión (11) se puede calcular la precisión de determinación de la peor solución entre las $\alpha$-óptimas encontradas; una vez obtenida la precisión requerida son seleccionadas las soluciones que se diferencian de la óptima en no más que $\alpha$.

\section{Consideración de la no-unicidad de algunas propuestas}

La serie (1) caracteriza distintas soluciones de una subtarea, pero con frecuencia ocurre que se obtienen soluciones múltiples con el mismo valor de la función de pérdidas locales, aspecto que puede ser analizado a partir de que cada propuesta se refiere no solo a una solución, sino a un espacio de éstas.

O sea, pueden existir casos de no-unicidad de los vectores de los parámetros de estado correspondientes a determinados miembros de dicha serie. Para estos casos, en vez de calcular directamente la función de pérdidas sistémicas para la combinación señalada, surge la tarea de optimización:

$$
\varepsilon\left(e^{k}\right)=\min \left\{\varepsilon\left(x_{k}\right) / g_{r}\left(x_{k}\right) \geq 0, r \in \overline{(1, m)} ; x_{k} \in X_{k}\right\}
$$

donde $X_{k}$ es el espacio de los vectores de estado correspondiente a la combinación de soluciones locales $e^{k}$.

Al ser la dimensión de las tareas(12) mucho menor que la de la tarea original, el algoritmo de selección óptima conduce a su descomposición en una serie de tareas más sencillas. 


\section{Función de pérdidas sistémicas generalizada}

En la formulación original de la tarea de Selección de Propuestas se parte del supuesto que la función de pérdidas sistémicas cumple con el requisito: $\varepsilon\left(e^{k}\right) \geq 0$. Este requisito, aunque muy general, puede no ser satisfecho en muchas tareas concretas. No obstante, como se verá a continuación, no ofrece mayores dificultades la generalización de la clase de funciones $\varepsilon\left(e^{k}\right)$ posibles a ser utilizadas en las tareas de Selección de Propuestas que admiten formulación y solución.

Sea $\varepsilon\left(e^{k}\right)$ tal que satisface el requisito general:

$$
-\infty<\varepsilon^{-} \leq \varepsilon\left(e^{k}\right) \leq+\infty
$$

La función de pérdidas sistémicas definida por la expresión (13) tiene como único requisito estar acotada inferiormente. Ella puede ser calculada por cualquier expresión analítica o procedimiento de cálculo.

Sea la función auxiliar:

$$
\varepsilon^{\mid}\left(e^{k}\right)=\varepsilon\left(e^{k}\right)-\varepsilon^{-} \geq 0
$$

La tarea original (4) es equivalente a la minimización de:

$$
Z\left(e^{k}\right)=\sum_{s=1}^{n} Z_{k}^{s}+\varepsilon\left(e^{k}\right) / e^{k} \in E
$$

donde $\varepsilon\left(e^{k}\right)$ está definido por la relación (13).

La nueva tarea de Selección de Propuestas reformulada es, evidentemente, equivalente a:

$$
\min _{e^{k}} Z\left(e^{k}\right)=\min _{e^{k}}\left\{\sum_{s=1}^{n} Z_{k}^{s}+\varepsilon^{\prime}\left(e^{k}\right) / e^{k} \in E\right\}+\varepsilon^{-}
$$

Así, la solución a la tarea (4) es la solución a la tarea (15), siempre que se utilice, en calidad de pérdidas sistémicas, la función $\varepsilon^{\prime}\left(e^{k}\right)$. Esto último equivale a incrementar $\varepsilon^{-}$en los términos a la izquierda en las expresiones (5) y (6) como nuevos requisitos para la eliminación de nodos y propuestas respectivamente, para la tarea reformulada de Selección de Propuestas, adoptando la función de pérdidas sistémicas generalizada según la expresión (13). La definición de una cota inferior para la función de pérdidas sistémicas puede contribuir significativamente a la reducción de la cantidad de iteraciones y de nodos generados por el algoritmo de solución, según se puede observar en los resultados del próximo acápite.

\section{La Tarea de Selección de Propuestas en la descomposición de tareas com- plejas de optimización discreta}

Las tareas de dirección, en ocasiones, alcanzan un alto nivel de complejidad debido entre otros factores a la gran cantidad de variables y restricciones que son necesarias analizar; de esta manera es que con el objetivo de hacer accesible encontrar la solución de problemas concretos, tiende a simplificarse este, para lo cual se adoptan, en general, modelos lineales. 
El método de Selección de Propuestas utilizado en combinación con el método aproximatorio-combinatorio en casos de tareas complejas de optimización discreta, conduce a algoritmos, los cuales pueden demostrar su eficiencia y son relativamente sencillos.

El esquema de descomposición es el siguiente:

Problema 5 Hallar la solución de la tarea:

$$
\begin{aligned}
& Z=\min \left\{f(x) / g_{0 k}(x) \geq b_{k}, k=1, \ldots, m\right\} \\
& x \geq 0 ; \quad x=\left(x_{1}, x_{2}, \ldots, x_{q}\right) \in D
\end{aligned}
$$

donde $D$ es un conjunto discreto de puntos.

Si las funciones $g_{0 k}(x)$ son separables con respecto a los vectores $x_{s}$ que forman una partición del vector $x$, este puede ser representado por:

$$
x=\left(x_{1}, \ldots, x_{2}, \ldots, x_{n}\right)
$$

donde

$$
\begin{aligned}
x_{s} & =\left(x_{i_{1}}, \ldots, x_{i_{j}}, \ldots, x_{i_{j_{s}}}\right) \\
i_{j} & \in W_{s} \subset W=(1,2, \ldots, q) \\
W & =\bigcup_{s} W_{s}, \bigcap_{s} W_{s}=\emptyset \\
g_{0 k}(x) & =\sum_{s} g_{s k}\left(x_{s}\right) \geq b_{k}, \quad k=1,2, \ldots, m
\end{aligned}
$$

De esta forma, cada una de las variables originales forma parte de uno y solo uno de los $n$ vectores $(n<q)$ en los que se particiona el vector $x$.

Sea, además, la función $f(x)$ representable en la forma:

$$
f(x)=\sum_{s} f_{s}\left(x_{s}\right)+\varepsilon(x) ; \quad \varepsilon(x) \geq 0 .
$$

Partiendo de que la función objetivo puede ser representada como la suma de $n$ funciones de los respectivos vectores que conforman la partición del vector $x$ más una función que adopta sólo valores positivos (generalmente, puede hacerse con ayuda de funciones aproximatorias).

Al realizar las sustituciones, la tarea (16) queda de la siguiente forma:

$$
\begin{aligned}
Z & =\min _{x_{s}} \sum_{s} f_{s}\left(x_{s}\right)+\varepsilon(x) \\
g_{k s}\left(x_{s}\right) & =y_{s k} ; \sum_{s} y_{s k} \geq b_{k}, \quad k \in(1, \ldots, m) \\
x_{s} & \geq 0, x_{s} \in D_{s}, \forall s \in S
\end{aligned}
$$

La tarea (19) se pone en correspondencia con una tarea coordinadora:

$$
\begin{aligned}
Z & =\min _{y}\{F(y)+\varepsilon(x)\} \\
\sum_{s} y_{s k} & \geq b_{k} ; \quad k \in(1, \ldots, m)
\end{aligned}
$$


que es análoga al centro en un sistema jerárquico de dos niveles.

El papel de los subsistemas del nivel inferior en los sistemas jerárquicos, por analogía, lo desempeñan las $n$ tareas locales:

$$
\begin{aligned}
Z^{s} & =\min f_{s}\left(x_{s}\right), \\
g_{s k}\left(x_{s}\right) & =y_{s k} ; \quad x_{s} \geq 0, x \in D_{s}
\end{aligned}
$$

Los valores óptimos de los criterios de optimilidad de las tareas locales (21) son funciones de las magnitudes $y_{s k}$.

La función $F(y)$ de la tarea (20) es definida por:

$$
F(y)=\sum_{s} Z^{s}\left(y_{s k}\right)
$$

El signo de las variables $y_{s k}$ no se restringe en la tarea (21), pues pueden ser interpretadas como el consumo del recurso $k$ o su producción. A pesar de esto, el objetivo es la descomposición del vector $x$ en subtareas independientes entre sí y que sea posible la separación entre los productos consumidos y producidos.

A partir de las condiciones de la tarea es posible determinar el signo de $y_{s k}$, al efectuar la sustitución:

$$
u_{s k}=\theta_{s k} y_{s k}
$$

$\theta_{s k}=\left\{\begin{array}{l}1 \text { si por el sentido de la restricción } k \text { de la tareas, } y_{s k} \text { es una magnitud positiva } \\ -1 \text { en caso contrario }\end{array}\right.$

La tarea (20) se convierte en :

$$
\begin{aligned}
Z & =\min _{u, x}\{F(u)+\varepsilon(x)\} \\
\sum_{s} \theta_{s k} u_{s k} & \geq b_{k}, k \in(1, \ldots, m) \\
u_{s k} & \geq 0, k \in(1, \ldots, m)
\end{aligned}
$$

Las restricciones de la tarea (24) definen una región $U$ denominada región de directivas posibles, tomando las tareas locales la forma:

$$
\begin{aligned}
Z^{s} & =\min _{u, x}\left\{f_{s}\left(x_{s}\right)\right. \\
\theta_{s k} g_{s k}\left(x_{s}\right) & =u_{s k}, \quad k \in(1, \cdots m) \\
x_{s} & \geq 0, \quad x_{s} \in D_{s}
\end{aligned}
$$

Para resolver las tareas (24) y (25) se necesita:

1. Hallar una función $Q(u)$, la cual se define en la región $U$ y un parámetro $\alpha \geq 0$, para los cuales se cumple:

$$
\min _{u} Q(u) \leq \min _{u, x}\{F(u)+\varepsilon(x)\} \leq \min _{u} Q(u)+\alpha
$$

La utilización de modelos de programación en enteros para la definición de $Q(u)$, en ocasiones resulta suficiente, por ejemplo, se puede adoptar:

$$
Q(u) \leq F(u)+\varepsilon(x), \forall u \in U, x \in D
$$


2. Solucionar la tarea

$$
Q(u) \leq \min _{u}\{Q(u) / u \in U\}+\alpha
$$

Esto equivale a hallar la serie de soluciones $\alpha$-óptimas de la función objetivo de la tarea coordinadora en la región $U$ :

$$
Q\left(u^{1}\right)<Q\left(u^{2}\right)<\ldots<Q\left(u^{i^{*}}\right) / Q\left(u^{i}\right)-Q\left(u^{1}\right) \leq \alpha
$$

a la que corresponde el conjunto de índices:

$$
I_{0}=\left(1,2, \ldots, i^{*}\right)
$$

3. Determinar la solución $u^{k}$ para la cual el conjunto de soluciones de la tarea (24) es tal que:

$$
\begin{aligned}
\sum_{s} Z^{s}\left(u_{s}^{k}\right)+\varepsilon\left(x^{k}\right) & \leq \sum_{s} Z^{s}\left(u_{s}\right)+\varepsilon(x) \\
k & \in I_{0}, u \in U, x \in D
\end{aligned}
$$

Para cada uno de los $u^{i}, i \in I_{0}$ se busca solución a la tarea:

$$
\sum_{s} Z^{s}\left(u_{s}^{k}\right)+\varepsilon\left(x^{k}\right) \leq \operatorname{mín}\left\{\sum_{s} Z^{s}\left(u_{s}^{i}\right)+\varepsilon(x)\right\}
$$

mediante el algoritmo de selección óptima entre las soluciones $\alpha$-òptimas de la familia de tareas (27).

La utilización del método aproximado-combinatorio (Jachaturov, 1989), permite hallar la solución óptima de la tarea coordinadora $u^{k}$, para la cual se cumple (30):

$$
\sum_{s} Z^{s}\left(u_{s}^{k}\right)+\varepsilon\left(x^{k}\right) \leq \min _{i \in I_{0}}\left\{Z^{s}\left(u_{s}^{i}\right)+\varepsilon\left(x^{i}\right)\right\}
$$

Si la función $Q(u)$ se define por (27), la búsqueda de la solución óptima se puede detener cuando se cumpla:

$$
Q\left(u^{i}\right)>\min _{k=1, \ldots, i-1}\left\{\sum_{s} Z^{s}\left(u_{s}^{k}\right)+\varepsilon\left(x^{k}\right)\right\}
$$

La expresión (33) es justificada por el Teorema 1, a partir de considerar a $Q(u)$ como función de pérdidas locales para un único elemento del sistema y $F(u)+\varepsilon(x)$ como función de pérdidas totales de sistema, siendo en este caso, la solución óptima de las tareas coordinadoras $u^{k^{*}}$ donde $k^{*}$ es determinada a partir de:

$$
k^{*}=\arg \left\{\min _{k=1, \ldots, i-1}\left[F\left(u^{k}\right)+\varepsilon\left(x^{k}\right)\right]\right\}
$$




\section{Velocidad de convergencia del algoritmo de selección óptima}

La efectividad del algoritmo de búsqueda de la selección óptima de la tarea de selección de propuestas depende de la velocidad de crecimiento de $Z_{i}^{s}$, del parámetro $\alpha$, del carácter de la función de pérdidas sistémicas $\varepsilon\left(e^{k}\right)$, de la cota inferior $\varepsilon_{0}$ de las pérdidas sistémicas y de la compatibilización de las regiones de existencia de las soluciones locales (Fiol, 1989). De ser posible conocer o hallar al menos una solución posible $e^{m}$, entonces la eliminación de las ramificaciones se garantiza mediante (5), además la condición (7) limita la cantidad de soluciones locales necesarias, en lo que (8) evalúa la desviación de la solución obtenida con respecto al óptimo.

El estudio de la velocidad de convergencia, se realiza a partir de la experimentación del Algoritmo de Selección de Propuestas para diferentes valores de los parámetros y el procesamiento posterior de los resultados. Se utiliza la siguiente simbología:

Inter: Cantidad de iteraciones requeridas para hallar todas las soluciones $\alpha$-óptimas NO: Cantidad máxima de nodos en espera alcanzada en el proceso de búsqueda de las soluciones $\alpha$-óptimas.

Se realizó un diseño experimental $3^{k}$ y fueron analizados 5 factores con la siguiente designación:

- $\mathbf{N}$ : Número de elementos del sistema

- M : Primera iteración finita

- ALFEO : Diferencia entre $\alpha$ y el valor de la cota inferior de las pérdidas sistémicas $\left(\varepsilon_{0}\right)$

- ZM : Incremento promedio de la función objetivo

- ZP : Valor inferior de las pérdidas locales

\section{Ecuación de regresión obtenida para la cantidad de iteraciones}

A continuación se muestran los resultados de un modelo de la regresión lineal múlti-

ple para describir la relación entre ITER(Iteraciones) y las 5 variables independientes utilizadas.

$$
\begin{aligned}
\text { ITER }= & 75,6719+24,2247 * N-10,1915 * N * Z M-0,195487 * M-14,8727 * Z P \\
& +1,02768 * N * M+2,33476 * N * A L F E O-, 337742 * A L F E O * Z P \\
& +2,18609 * Z P * Z M
\end{aligned}
$$

Como el valor de $P$ en la tabla ANOVA es menor que 0.01, hay una relación estadísticamente significativa entre las variables a un $99 \%$ de nivel de confianza.

El parámetro $R$ - cuadrado indica como se ajustó el modelo con un $99.6921 \%$ de variabilidad en la variable ITER.

El error standard de la estimación muestra la desviación normal de los residuos que es de 14.1882 .

El error de estimación de ITER calculado por la ecuación (35), para un intervalo de confianza del $95 \%$ es de $\pm 28,3764$. 
El error absoluto medio es de 10.1921 y representa el valor medio de los residuos.

El coeficiente de Durbin-Watson (DW) en las pruebas estadísticas de los residuos sirve para determinar si hay cualquier relación significante en la correlación basada en el orden en el que ellos ocurren en el archivo de los datos. Como el valor de DW es mayor que 1.4, no hay probablemente cualquier autocorrelación seria en los residuos.

La tabla de los residuos inusuales lista todas las observaciones que tienen residuos Studentizados mayor que 2.0 en valor absoluto. En este caso, hay 4 residuos Studentizados mayores que 2.0, pero ninguno mayor que 3.0.

La tabla de los datos influyentes presenta todas las observaciones que tienen influencia en su valor mayor que 3 veces el del valor medio de los datos, o que tiene un valor grande de DFITS. La influencia es una estadística que mide cómo influye cada observación en la determinación de los coeficientes del modelo estimado. DFITS es una técnica estadística que mide cuánto los estimadores de los coeficientes cambiarían si cada observación era quitada del juego de los datos. En este caso, el valor de influencia es igual a 0.152542 . No hay ningún punto de los datos con más de 3 veces la media influencia.

El análisis de los resultados permite obtener la ecuación (2.40) como una aproximación para analizar la influencia de los diferentes factores predeterminados sobre la cantidad de iteraciones realizadas, pues es evidente que mientras mayor sea el número de iteraciones, mayor será la demora en la ejecución del programa; es importante destacar que, con los datos obtenidos, se logra que el crecimiento de las iteraciones no sea exponencial, resaltándose la influencia, entre otros factores, de la cantidad de elementos del sistema y del valor inferior de las pérdidas locales.

\section{Ecuación de regresión obtenida para la cantidad de nodos}

A continuación se muestran los resultados de un modelo de la regresión lineal múltiple para describir la relación entre $\mathbf{N o ( n o d o s )}$ y las 5 variables independientes.

La ecuación del modelo ajustado es

$$
\begin{aligned}
N O= & 33,0602+22,0629 * N-7,04009 * N * Z M-1,75511 * M-7,25902 * Z P \\
& +0,87085 * N * M+1,30528 * N * A L F E O \\
& -0,218987 * A L F E O * Z P+1,37699 * Z P * Z M
\end{aligned}
$$

Como el valor de $\mathbf{P}$ en la tabla ANOVA es menor que 0.01 , hay una relación estadísticamente significante entre las variables a un $99 \%$ de nivel de confianza.

El parámetro R-cuadrado indica como se ajustó el modelo con un $99.3646 \%$ de variabilidad en la variable NO.

El error estándar de la estimación muestra la desviación normal de los residuos, que es de 7.39584.

El error de estimación de NO calculado por la ecuación (36), para un intervalo de confianza del $95 \%$ es de $\pm 14,7917$

El error absuluto medio de 5.18294 representa el valor medio de los residuos.

El coeficiente de Durbin- Watson (DW) en las pruebas estadísticas de los residuos sirve para determinar si hay cualquier relación significante en la correlación basada en el orden en el que ellos ocurren en su archivo de los datos. Como el valor de DW es mayor que 1.4, no hay probablemente cualquier autocorrelación seria en los residuos. 
La tabla de los residuos unusuales lista todas las observaciones que tienen residuos Studentizados mayor que 2.0 en valor absoluto. En este caso, hay 4 residuos Studentizados mayores que 2.0, pero ninguno mayor que 3.0.

La tabla de los datos influyentes presenta todas las observaciones que tienen influencia en su valor mayor que 3 veces el del valor medio de los datos, o que tiene un valor grande de DFITS. La influencia es una estadística que mide cómo influye cada observación en la determinación de los coeficientes del modelo estimado. DFITSes una técnica estadística que mide cuánto los estimadores de los coeficientes cambiarían si cada observación era quitada del juego de los datos. En este caso, el valor de influencia es igual a 0.155172 . No hay ningún punto de los datos con más de 3 veces la media influencia.

El análisis de los resultados permite obtener la ecuación (36) como una aproximación para analizar la influencia de los diferentes factores predeterminados sobre la cantidad de nodos, pues es obvio que un número elevado de nodos, aumentaría en grado considerable la ejecución del programa; es bueno destacar que, con los datos obtenidos, se logra que el crecimiento de los nodos no sea exponencial, destacándose la influencia de la cantidad de elementos del sistema.

Con vistas a ampliar el conjunto de problemas prácticos a enfrentar se desarrolló la siguiente Tarea Generalizada de Selección de Propuestas.

\section{Tarea Generalizada de Selección de Propuestas}

En no todas las aplicaciones es posible separar la función de pérdidas locales para cada subtarea y para la tarea total, así como estimar un valor de la cota de la función de pérdidas sistémicas lo suficientemente próxima a su valor real. De aquí la conveniencia potencial que tendría la posibilidad de generar propuestas no evaluadas previamente, lo que conduce a la siguiente formulación.

Sea cada elemento del conjunto finito $S=(1,2, \ldots, s, \ldots, n)$ puesto en correspondencia con una serie ordenada, monótona creciente de números enteros:

$$
I_{s}=(1,2, \ldots, i, \ldots), \forall s \in S
$$

La serie (5) representa diferentes opciones de solución a cada una de las subtareas $s \in S$.

Las combinaciones posibles elementos de los conjuntos (37) se determinan por los elementos

$$
e^{k}=\left(k_{1}, k_{2}, \ldots, k_{s}, \ldots, k_{n}\right)
$$

del producto de conjuntos:

$$
E=I_{1} \times I_{2} \times \ldots \times I_{n}
$$

Para cada $e^{k} \in E$, se requiere minimizar

$$
Z\left(e^{k}\right) / e^{k} \in E
$$

asegurando el cumplimiento de un conjunto de restricciones:

$$
g_{j}\left(e^{k}\right) \geq b_{j} ; \quad \forall j=1, \ldots, m
$$

Si en la tarea (37)-(39) los elementos de cada uno de los conjuntos $I_{s}$ se consideran enumeraciones de los diferentes valores de las variables $x_{s} \in X_{s}, \forall s \in S$, los elementos 
$e^{k} \in E$ constituyen entonces enumeraciones de los diferentes valores del vector de variables del problema $x \in X=X_{1} \times X_{2} \times \ldots \times X_{n}$. En este caso, la tarea (37)-(39) es equivalente a la siguiente:

Minimizar:

$$
Z(x) / x \in X
$$

asegurando:

$$
g_{j}(x) \geq b_{j} ; \quad \forall j=1, \ldots, m .
$$

La tarea (40)-(41) no es otra cosa que la tarea general de la programación discreta.

La interpretación de (37)-(39) puede, sin embargo, ser examinada de otra forma: los elementos del conjunto $I_{s}$ pueden constituir diferentes opciones de solución de subtareas de un problema de optimización discreta.

$$
\begin{aligned}
Z & =\min \left\{Z(x) / g_{j}(x) \geq b_{j}, \quad j=1, \ldots, m\right\} \\
x & =\left(x_{1}, x_{2}, \ldots, x_{q}\right) \in X
\end{aligned}
$$

donde $X$ es un conjunto discreto de puntos.

Si el conjunto $X$ se particiona en una serie de subconjuntos, cada uno de los cuales agrupa los valores del espacio de definición de una parte de las variables de la tarea (42), es decir,

$$
x=\left(x_{1}, \ldots, x_{s}, \ldots, x_{n}\right)
$$

donde

$$
\begin{gathered}
x_{s}=\left(x_{i_{1}}, \ldots, x_{i_{j}}, \ldots, x_{i_{j_{s}}}\right) \\
i_{j} \in W_{s} \subset W=(1,2, \ldots, q) \\
\bigcup_{s} W_{s}=W, \quad \bigcap_{s} W_{s}=\emptyset
\end{gathered}
$$

entonces, la tarea (42) se descompone en una serie de subtareas fuertemente vinculadas entre sí, tanto por la función objetivo como por todas y cada una de las restricciones.

Ahora, si por algún procedimiento se seleccionan, por el decisor correspondiente, aquellas soluciones que resultan de interés en cada subespacio de soluciones $X_{s}$ (por ejemplo, con ayuda del método aproximatorio-combinatorio), y se les ordena por algún criterio cuantificable o subjetivo, entonces, la enumeración de estas soluciones constituyen los conjuntos $I_{s}$ definidos por (37). El producto cartesiano de los conjuntos $I_{s}$ constituye el conjunto $E$, el que no es otra cosa que las posibles soluciones seleccionadas y enumeradas por el conjunto de decisores $S$. En estas condiciones, la función objetivo de la tarea original $Z(x)$ adopta la forma $Z\left(e^{k}\right)$.

Así, la Tarea Generalizada de Selección de Propuestas no es otra cosa que la tarea general de la programación discreta cuando por algún procedimiento el (o los) decisores particionan el conjunto de soluciones admisibles en un grupo de subconjuntos y de cada uno de ellos seleccionan y ordenan de acuerdo a un criterio de preferencia (objetivo o subjetivo) algunas de las soluciones de cada subconjunto, y buscan solución al problema general entre las diferentes combinaciones de opciones seleccionadas. En la ponencia se brinda algoritmo de solución de la tarea generalizada de Selección de Propuestas basado en los Algoritmos Genéticos. 


\section{Enfoques para la conciliación de decisiones entre sistemas organizados en diferentes estructuras}

La conciliación de decisiones de sistemas interrelacionados requiere de la organización de determinadas estructuras entre los sistemas. Son posibles las siguientes estructuras de conciliación:

\section{- Estructura disperso conciliada.}

En esta estructura cada sistema prepara sus decisiones de forma independiente, suministrando a los restantes sistemas del mismo nivel funciones aproximatorias que expresan la influencia de las variables de decisión de otros sistemas sobre sus propios indicadores de eficiencia. Se establecen restricciones en cada sistema a la afectación provocada a los restantes sistemas. No existe relación de subordinación entre los sistemas. Esta estructura resulta muy frecuentemente apropiada a los sistemas de preparación de decisiones relacionados con el diseño de tecnologías.

\section{- Estructura centralizada.}

En esta estructura el sistema de mayor nivel elabora acciones directivas que son de obligatorio cumplimiento para los sistemas del nivel inferior. Para un funcionamiento eficiente de esta estructura se requiere de la utilización de funciones aproximatorias de las acciones óptimas de los sistemas del nivel inferior, dependientes de la acción directiva del nivel superior. Este tipo de estructura resulta muy frecuentemente apropiada para los sistemas de control de procesos, en los que el tiempo de preparación de decisiones resulta muy limitado.

\section{- Estructura jerárquica.}

En esta estructura el sistema del nivel superior elabora acciones directivas, generalizadoras del funcionamiento de todo el sistema, a cada sistema del nivel inferior, cada uno de estos últimos elabora alternativas de cumplimiento de la acción recibida, ordenadas de acuerdo, a un criterio local de eficiencia, entregando estas opciones, en calidad de propuestas, al sistema del nivel superior. En una tercera etapa, el sistema del nivel superior selecciona una combinación de propuestas de los sistemas del nivel inferior que satisface, de la mejor manera (optimiza) un criterio de eficiencia generalizador para todo el sistema. Este tipo de estructura resulta muy frecuentemente apropiada para los sistemas de carácter organizativo.

La Tarea de Selección de Propuestas está concebida, precisamente, para dar solución a la tercera etapa de funcionamiento de la estructura jerárquica: la etapa de toma decisiones, siendo las dos etapas anteriores de preparación de decisiones.

Cada una de las estructuras de conciliación de decisiones entre sistemas enumeradas se corresponde con respectivos esquemas de descomposición de tareas de optimización y de composición posterior de soluciones entre las subtareas resultantes. El autor ha estudiado los enfoques adecuados de solución para cada caso.

\section{Aplicaciones realizadas}

El método de Selección de Propuestas y los esquemas expuestos de descomposición de tareas de optimización discreta de gran complejidad han sido aplicados por el autor a la 
solución de las siguientes tareas prácticas:

- Elaboración de gráficos intercambio de cilindros en trenes de laminación de perfiles ligeros (Arzola, 1989).

- Conciliación de secuencias de trabajo de objetos productivos que trabajan en paralelo (Arzola, 1989).

- Diseño óptimo multiobjeto de redes de conductos de climatización (Cordovés, 1999).

- Generación de tecnologías de maquinado en tornos con mando numérico computarizado (Ávila, 1999).

- Diseño óptimo multiobjetivo de troqueles de corte y punzonado simples y progresivos (Simeón, 1999).

\section{Referencias}

[1] Arzola, J. (1989) Selección de Propuestas. Editorial Científico Técnica, La Habana.

[2] Arzola, J. (2000) Sistemas de ingeniería. Editorial Félix Varela, La Habana.

[3] Arzola, J.; Suárez, L. (1993) "Reglas de conducta en la proyección y conducción de procesos de calentamiento del acero", Rev. Argus, Saltillo (México).

[4] Ávila, R. (1999) Generación de Tecnología para Máquinas Herramienta con Control Numérico Computarizado. Tesis doctoral en Ciencias Técnicas, Holguín.

[5] Cordovés, A.(1999) Diseño Óptimo Multiobjetivo y Preparación para la Fabricación (CAD/CAPP) de Redes de Conductos de Climatización. Tesis doctoral en Ciencias Técnicas, Holguín.

[6] Cordovés, A.; Arzola, J. (1999) "Diseño óptimo multiobjetivo y preparación para la fabricación $(\mathrm{CAD} / \mathrm{CaPP})$ de redes de conductos de climatización", Ingeniería Mecánica 2(1).

[7] Díaz, A y otros (1996) Optimización Heurística y Redes Neuronales. Editorial Paraninfo, Madrid.

[8] Fiol, A. (1999) El Método de Selección de Propuestas y su Generalización. Tesis de Maestría en Optimización y Toma de Decisiones, ISPJAE, La Habana.

[9] Jachaturov, V. Métodos Matemáticos de la Programación Regional. Editorial Naúka, Moscú.

[10] Komoda, N.; Kera, K.; Kubo, T.(1984) Computer 17 (12): 46-51.

[11] Lewandowski, A.; Wierzbicki, A. (1989) Aspiration Based Decision Support Systems. SpringerVerlag, Berlin.

[12] Mesarovic, M.; Macko, D.; Takahara, Y. (1970) Theory of Hierarhial, Multilevel Systems. Academic Press, New York-London.

[13] Simeón, R. (1999) Diseño Óptimo Multiobjetivo de Troqueles de Corte y Punzonado Simples y Progresivos. Tesis doctoral en Ciencias Técnicas, Holguín.

[14] Wiener, N. (1949) Cibernetics, or Control and Communication in the Animal and Machine. John Wiley \& Sons, New York. 